\title{
DEUX HYDROPSYCHE CAUCASIENS NOUVEAUX DU GROUPE \\ FULVIPES-INSTABILIS \\ [Trichoptera]
}

par L. Botosaneanu.

Peu de temps avant sa mort ${ }^{1}, \mathbf{M}^{\text {me }}$ S. G. Lepneva m'envoya une séric de 9 exemplaires d'Hydropsyche du Caucase, en m'invitant d'en entreprendre l'étude. L'examen de cette petite collection m'a montré qu'elle se composait surtout d'espèces du groupe fulvipesinstabilis, dont deux nouvelles; mais il n'était plus possible de décrire ces espèces dans le travail consacré aux Hydropsyche de ce groupe [Botosaneanu et Marinkovic-Gospodnetic 1966], travail qui était déjà sous presse. Je le ferai donc maintenant.

La collection comprend les éléments suivants :

1. Deux of des environ d'Ordjonikidze (Vladycaucase), de la collection Mar'rynov, capturés par Ryabov et accompagnés par des étiquettes de la main de MarTynov. Il s'agit d'Hydropsyche martynovi n. sp.

2. Deux ô capturés par Satunin en Adjarie, de la collection Martynov et pourvus, tous les deux, d'étiquettes identiques de sa main. L'un représente le type d'Hydropsyche lepnevae n. sp., l'autre est un $H$. consanguinea McL. Deux exemplaires $\$$, accompagnés par des étiquettes identiques, n'ont pas été étudiés.

3. Trois o de Bakuriani, Gruzie (ruisseau Bakurianka, 20.VII.1953 et 11.VIII.1953, leg. I. Zhiltzova et A. Cistiakova) sont des H. mahrkusha Schmid. Deux de ces exemplaires ont une envergure de $19 \mathrm{~mm}$, l'envergure du troisième est de $17 \mathrm{~mm}$; ils paraissent se distinguer des exemplaires iraniens connus par certains détails des armatures génitales (l'espèce semble assez variable).

Hydropsyche lepnevae. n. sp. (fig. 1).

Envergure, holotype $\delta: 19,5 \mathrm{~mm}$. Ailes antérieures d'un jaune paille très clair; la pilosité en est extrêmement réduite; je pense

1. Mme S. G. Lepneva, éminente spécialiste des Trichoptères et limnologue de Léningrad, s'est éteinte le 8 octobre 1966, à l'âge de 83 ans. 

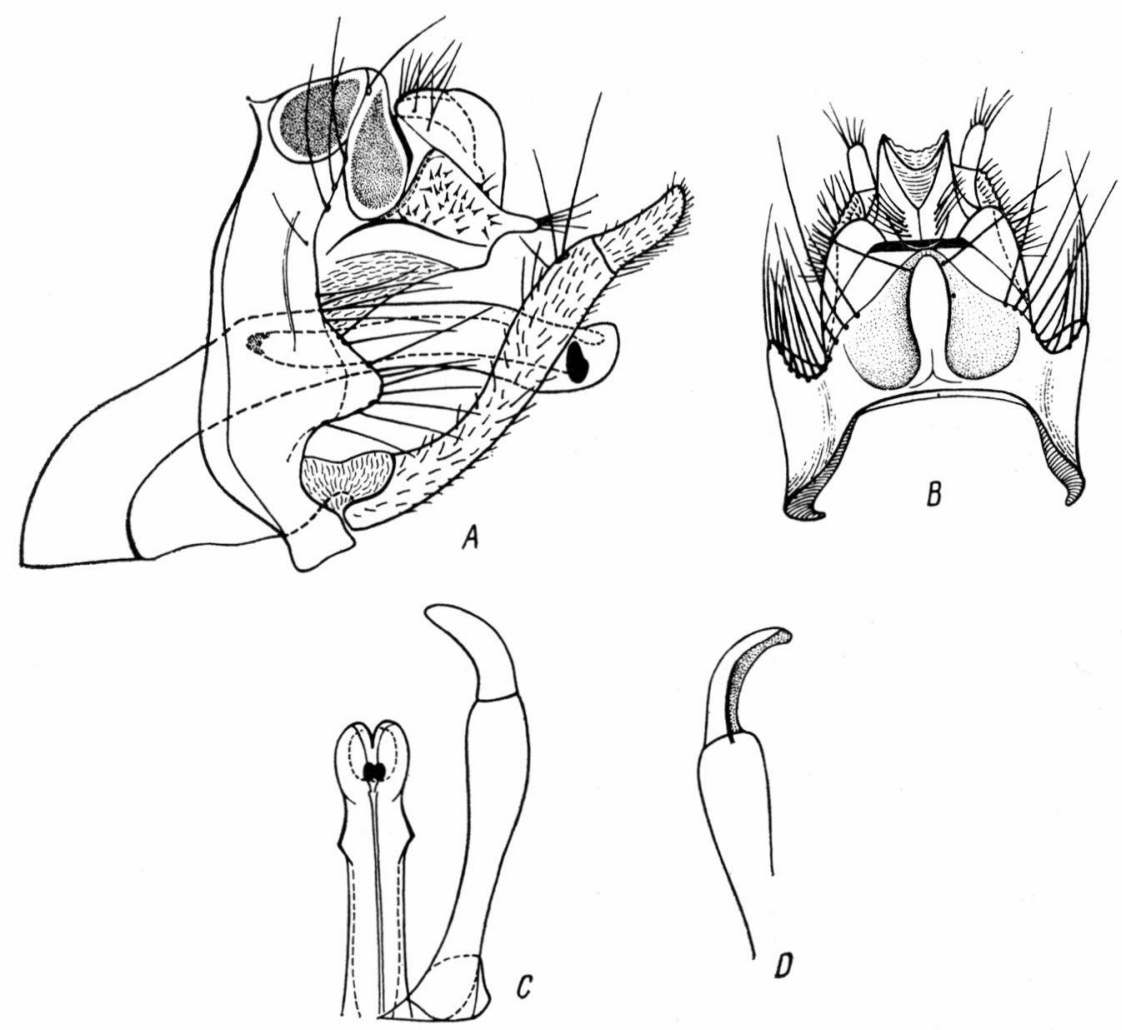

Fig. 1. - Hydropsyche lepnevae n. sp. Holotype $\sigma^{-}$, armature génitale. A : later.; B. : dors; C. : gonopode gauche et phallus, ventr.; D : face dorsale du gonopode.

que c'est l'espèce la plus pâle du groupe. Les antennes de l'exemplaire sont brisées. Tête et thorax d'un brun-pâle, avec un reflet rougeâtre. Armature génitale très claire.

Segment IX étroit latéralement. Car.d.IX $X^{1}$ parabolique à l'apex, les bords légèrement arqués (paraissant parfois même parallèles), la partie proximale nettement plus étroite formant comme un manche. Lim.d.cav.IX montant faiblement vers le haut. Les deux cavités (IX et $\mathrm{X}$ ) ne sont pas très profondes (à peu près de la même profondeur toutes les deux); elles sont distinctes, nettement séparées par lim.IX/X; les dimensions de cavit. $X$ sont légèrement plus grandes que celles de cavit.d.IX; en vue dorsale, les cavit.d.IX sont

1. Pour les abréviations utilisées, voir Botosaneanu et MarinkovicGospodnetic [1966]. 
arrondies; leur limite proximale est nettement arrondie en vue latérale et largement détachée de la limite proximale du segment. Observer l'aspect angulaire de la zone qui sépare, du côté ventral, les deux dépressions (lim.v.cav. $I X / X$ ) ; mais cet angle est en réalité un peu plus émoussé que ne le montre la figure $1 \mathrm{~A}$. Voir aussi le trait que nous avons figuré ( fig. $1 \mathrm{~A}$ ) comme continuant lim.v.z.spin. en direction de $\lim . I X / X$ - mais sans la rejoindre (ce trait prend - en vue dorsale, fig. 1 B. - l'aspect d'une infime « épaule 》 en bas de la cavit. $X$ respective). La limite anale de cavit. $X$ est formée par lim.p. (d.) z.spin. et par un carène qui part de cette limite pour finir au fond du sinus séparant (vue latérale, fig. 1 A) cavit.X de ail. p.X. En vue dorsale (fig. 1 B), le relief de la zone comprise entre centre $X$, la lim. dist. $z$. spin. et l'extrémité distale de cavit. $X$, sont assez compliquées et il est difficile de dire si la figure représente fidèlement la réalité. $B$. $t r$. très nette, ses prolongements sont euxaussi distincts, aussi bien en vue latérale, qu'en vue dorsale. $B$. $t r$. n'atteint pas lim. p. (d.) z. spin. qui se prolonge directement par lim. dist. z. spin; cette dernière est droite, très oblique vers l'arrière (on $\mathrm{y}$ aperçoit quelques verrues supportant des spinules) et elle trouve son prolongement direct dans la limite dorsale de l'app. digit. La lim. v. z. spin. devient fort indistincte vers son extrémité, elle n'atteint probablement pas le menton. App. digit. courts, il ne se prolongent pas derrière la $z$. spin. dont l'ampleur est assez réduite, avec des spinules assez rares; la lim. dist. z. spin., droite et oblique, lui donne un aspect « tronqué ». La partie dorsalement visible de $z$. spin. est très réduite, absolument latérale et apicale, non proéminente vers l'arrière, et coincée entre lim. dist. $z$. spin. et l'extrémité distale de cavit. $X$ (c'est cette cavité qui - fig. $1 \mathrm{~B}-$ cache des parties importantes de $z$. spin. - voir la limite en pointillé qui représente lim. $v$. $z$. spin. - sans pouvoir empêcher les longues spinules de déborder sur les flancs). Angle $\alpha$ de $90^{\circ}$. En prolongation de $b$. $t r$. se trouve un sinus profond et de forme caractéristique. Les deux ailettes de lim. centre $X$ sont indistinctement séparées, faiblement arrondies, la distale nettement mieux développée que la proximale. Il y a des soies seulement sur ail. $p . X$, quelques-unes descendent en direction ventrale. Lim. dist. $X$ descend directement à la racine de l'app. digit. Le centre $X$ est en ovale très allongé; une partie de ses limites latérales est difficilement distincte en vue dorsale, c'est pourquoi la figure $1 \mathrm{~B}$ ne représente pas cette partie. Golfe apic. extrêmement large et profond (semicirculaire), flanqué par des lobes très pointus. Menton proéminent. Épaules tombantes. Gonopodes graciles (ceci s'applique aussi bien au coxopodite qu'à l'harpago); l'harpago présente sur sa face médiane une concavité très nette, visible uniquement en position dorsale (fig. $1 \mathrm{D})$. Harp./cox. $=1 / 2,9$. Le phallus vu laté- 
ralement (fig. $1 \mathrm{~A}$ ) présente à la racine une courbure peu développée, on remarque une brusque flexion à peu près au milieu de la longueur, le gland n'est pas très nettement individualisé, l'apex se dirige vers le haut (fig. $1 \mathrm{C}) . A p . p h .(d .-v$.) est caractéristique avec ses dents phall. fortes mais proportionnées, pointues, légèrement déplacées du côté ventral, tandis que la partie apicale par rapport aux dents présente des bords nettement et régulièrement arrondis (convexes), cette partie du phallus étant presque circulaire.

Cette espèce s'apparente surtout probablement à tjederi Bots. et MARINK, mais aussi aux autres espèces voisines de tjederi (discreta, klefbecki, jordanensis).

Matériel étudié : 1 ô (holotype), 29.VII.1909, Adjaria-Caucase (sans localtié précisée), leg. Saturin. Martynov avait déterminé cet exemplaire comme «Hydropsyche instabilis CuRT. var.?». L'exemplaire (à sec, abdomen dans un petit tube, en alcool glycériné) est conservé dans le Musée Zoologique de Léningrad.

\section{Hydropsyche martynovi n. sp. (fig. 2).}

Envergure, holotype ô : $21 \mathrm{~mm}$. Ailes antérieures d'un brun-foncé d'un tonalité « chaude », à cause d'un reflet doré accentué; c'est une des espèces les plus foncées du groupe, sinon la plus foncée. Tête et thorax noirs. Armature génitale d'un brun-foncé ou très foncé, d'un noir brillant par endroits ( $z$. spin. plus pâle).

Segment IX large latéralement. Car. $d$. IX très large, paraissant bilobée à l'apex, sa surface recouverte de pointes minuscules, bords arrondis, largeur maximum au milieu, suture médiane très développée. En vue dorsale (fig. 2 B), l'apex de cav. d. IX s'applique presque contre $b$. $t r$. La $L i m$. $d$. cav. IX monte rapidement vers le haut. Cavit. $d$. IX en ovale régulier, assez large; contour de cavit. $X$ plutôt irrégulier; si le relief de la première est fort simple, la seconde a un relief assez compliqué. La $\operatorname{Lim}$. $I X / X$ ne sépare pas très nettement les deux cavités, surtout dans leur partie dorsale; au niveau intercavital, les soies ne sont pas insérées sur la limite même, mais légèrement déplacées du côté de cavit. $d . I X$; du côté de cavit. $X$, la limite est doublée par un rebord assez large et assez nettement distinct (même en vue dorsale, fig. $2 \mathrm{~B}$ ). L'aspect de lim. $v$. cav. $I X-X$ est «en pointe ». Toutes les limites figurées sur le segm. $X$ sont tranchantes, à l'exception de la zone située ventralement par rapport à $z$. spin. La cavit. $X$ est distinctement séparée en deux zones par $b$. tr. (large) et par le trait qui prolonge celle-ci; le fond proprement dit de la dépression est assez réduit; la zone distale par rapport à $b$. $t r$. et à son prolongement, forme une paroi inclinée, comme dans toutes les espèces, mais ici cette paroi est nettement séparée en deux zones par une ramiflcation venant de 


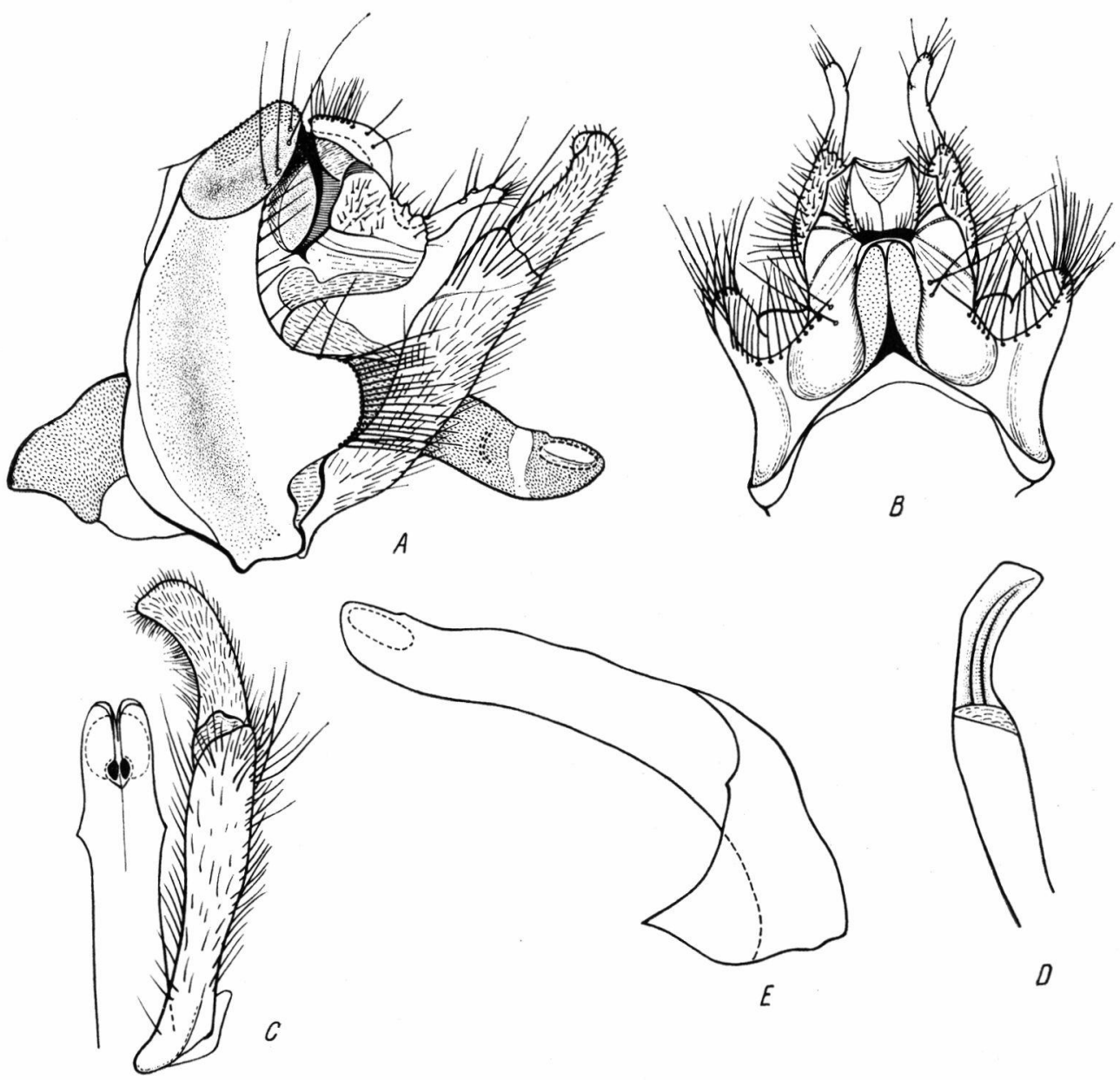

Fig. 2. - Hydropsyche martynovi n. sp. Holotype $\sigma^{*}$, armature génitale. A : later.; B : dors.; C : gonopode gauche et phallus, ventr.; D: face dorsale du gonopode.

b. $t r$. (fig. $2 \mathrm{~A}$ ) et ceci est visible aussi en position dorsale (fig. $2 \mathrm{~B}$ ). $Z$. spin. triangulaire (remarquer la petite zone en hachures à l'angle proximal-dorsal). Lim. p. (d.) z. spin. atteint presque lim. dist. z. spin., lim. v. z. spin aboutit au menton. La moitié ventrale de la lim. dist. z. spin. est ondulée, elle passe nettement par-dessus la racine des app. digit. Ceux-ci sont très longs, irréguliers (assez réguliers dans le paratype), pourvus non seulement des soies apicales mais aussi d'un certain nombre de verrues spinuligères. En vue dorsale les $z$. spin. sont grandes, proéminentes, placées très 
latéralement, proéminent de façon distincte à la partie distale, largement séparées du centre $X$, et ne permettant presque pas à la zone sous-jacente d'être aperçue. Angle $\alpha$ obtus; mais il y a ici un sinus très distinct, étroit, dans le prolongement de $b$. tr. (et séparant lim. d. cav. IX de lim. centre $X$ ). Cette dernière est descendante; il n'y a pas séparation en deux ailettes individualisées; les soies sont rigides, elles forment une sorte de peigne. Le centre $X$ est trapu; golfe apic. prenant l'aspect d'une large émargination. Lim. dist. $X$. descend verticalement; elle rencontre lim. dist. $z$. spin dans un point largement distancé de la racine des app. digit. Menton fuyant (assez proéminent dans le paratype). Epaules tombantes (voir fig. $1 \mathrm{~B}$ : ne pas tenir compte de z. spin.!). Gonopodes : harp./cox. $=1 / 2$; l'harpago est très fort, large et tronqué à l'apex, formant un « bec » peu accusé vers la ligne médiane, et pourvu sur la face dorsale d'une carène saillante et large ( fig. $2 \mathrm{D})$. Une membrane (le frein) relie le phallus au segm. X. L'Ap. ph. (d.-v) est pratiquement identique à celui de $H$. peristerica; mais, en vue latérale, le phallus, bien que toujours du «type pellucidula», se caractérise par la moindre courbure de la partie proximale, par une «bosse » dorsale accusée faisant suite à cette partie proximale, par la longueur moindre et par l'apex un peu moins pointu.

Cette espèce s'apparente surtout probablement à peristerica Bots. et Marink. et aussi à instabilis Curt.

Matériel étudié : 1 ô (holotype) et 1 \& (paratype), 30.IV.1921, Caucase (rivière Tere, environs de Ordjonikidze = l'ancien Vladycaucase), leg. Ryabov. Deux étiquettes de la main de Martynov portent les inscriptions suivantes : "Hydropsyche fulvipes McL. Martynov det. » et "Hydropsyche instabilis f. fulvipes? MarTYNOV ». Les deux exemplaires (à sec, abdomens dans de petits tubes d'alcool glycériné) sont conservés au Musée Zoologique de Léningrad.

Pour se rendre compte de l'évolution des opinions de Martynov concernant les Hydropsyche caucasiens du groupe fulvipes-instabilis, il faudra consulter ses travaux de 1909, 1926, 1927, 1934.

\section{RÉSUMÉ}

Description de deux nouvelles espèces caucasiennes d'Hydropsyche du groupe fulvipes-instabilis; $H$. lepnevae $\mathrm{n}$. sp. et $H$. martynovi n. sp. Ces descriptions complètent les données d'un travail antérieur [Botosaneasu et Marinkovic-Gospodnetic 1966]. 

TWO NEW HYDROPSYCHE OF THE FULVIPES-INSTABILIS GROEP
FROM THE CAUCASUS

[Trichoptera]

Description of two new Hydropsyche - species of the fulvipesinstabilis complex found in the Caucasus : $H$. lepnevae n. sp. and $H$. martynovi n. sp. These descriptions are a supplement to the findings of a former]y published paper [Botosaneand et Marinkovic-Gospodnetic: $1966]$.

\section{ZWEI NEUE KAUKASISCHE HYDROPSYCHE DER FULVIPES-INSTABILIS GRUPPE}

\section{[Trichoptera]}

Beschreibung zwei neuer kaukasisher Hydropsyche — Arten des fulvipes-instabilis Gruppe : H. lepnevae n. sp. und H. martynovi n. sp. Diese Beschreibungen vervollständigen die Angaben einer früheren Arbeit [Botosaneanu et Marin Kovic-Gospodnetic 1966].

\section{TRAVAUX CITÉS}

Botosaneanu (L.) et Marinkovic-Gospodnetic (M.). 1966. - Contribution à la connaissance des Hydropsyche du groupe fulvipes-instabilis. Etude des génitalia mâles (Trichoptera). Annls Limnol., 2 (3) : 503-525.

Martynov (A.). 1909. - Die Trichopteren des Kaukasus. Zool. Jb. Aöt. Syst. Geogr. Biol. Tiere, 27 : 509-558.

Martynov (A.). 1926. - To the knowledge of Trichoptera from central Kaukasus and their metamorphoses. Trav. Stn Biologique Caucase du nord, 5 (3) : 19-62.

Martynov (A.). 1927. - Supplementary notes on the Trichopterous fauna of the Caucasus. Russk, entom. Obozr., $21(1 / 2)$ : 119-127.

Marty nov (A.). 1934. - Ruceiniki. Trichoptera, Annulipalpia. I. Opred. po faune SSSR. Izd. Zool. Inst. A.N. S.S.S.R. 13, 343 p., Leningrad.

(Institutul de Speologie

«E. Racovitza », Bucarest.) 